\title{
Validation of risk prediction models for the development of HBV-related HCC: a retrospective multi-center 10-year follow- up cohort study
}

\author{
Yeon Seok Seo,"*, Byoung Kuk Jang ${ }^{2, *}$, Soon Ho Um¹, Jae Seok Hwang', Kwang- \\ Hyub Han ${ }^{3}$, Sang Gyune Kim ${ }^{4}$, Kwan Sik Lee ${ }^{3}$, Seung Up Kim³ ${ }^{3}$ Young Seok Kim ${ }^{4}$ \\ and Jung II Lee ${ }^{3}$ \\ ${ }^{1}$ Department of Internal Medicine, Korea University, Seoul, Korea \\ ${ }^{2}$ Department of Internal Medicine, Keimyung University School of Medicine, Daegu, Korea \\ ${ }^{3}$ Department of Internal Medicine, Institute of Gastroenterology, Yonsei University College of Medicine, Seoul, Korea \\ ${ }^{4}$ Department of Internal Medicine, Soonchunhyang University Hospital, Bucheon, Korea \\ *These authors contributed equally to this work \\ Correspondence to: Seung Up Kim, email: ksukorea@yuhs.ac \\ Young Seok Kim, email: liverkys@schmc.ac.kr \\ Jung II Lee, email: mdflorence@yuhs.ac
}

Keywords: hepatitis $B$, hepatocellular carcinoma, transient elastography, risk prediction, liver stiffness

Received: May 31, $2017 \quad$ Accepted: October 02, $2017 \quad$ Published: November 03, 2017

Copyright: Seo et al. This is an open-access article distributed under the terms of the Creative Commons Attribution License 3.0 (CC BY 3.0), which permits unrestricted use, distribution, and reproduction in any medium, provided the original author and source are credited.

\section{ABSTRACT}

Recently, modified REACH-B (mREACH-B) risk prediction model for hepatocellular carcinoma (HCC) development was proposed. We validated the accuracy of the mREACH-B model and compared its accuracy with those of other prediction models. Between 2006 and 2012, 1,241 patients with chronic hepatitis B (CHB) were recruited. All patients underwent transient elastography at enrollment. The median age of the study population (840 males, 401 females) was 49 years. The median PAGE-B, LSMHCC, and mREACH-B values were 10, 10, and 8, respectively. Among patients without cirrhosis $(n=940,75.7 \%)$, the median REACH-B value was 9. During the follow-up period (median 77.4 months), $66(5.3 \%)$ and $83(6.7 \%)$ patients developed HCC and liver-related events (LRE), respectively. Higher liver stiffness (LS) independently predicted HCC (hazard ratio $[H R]=1.047)$ and LRE development $(H R=1.047)$ (all $P<0.05$ ). The mREACH-B significantly predicted HCC (AUC $=0.824$ at 3-year and 0.750 at 5-year) and LRE development (AUC $=0.782$ at 3 -year and 0.739 at 5-year) (all $P<0.001$ ) and it performed similarly or significantly better than the PAGE-B and LSM-HCC (AUC = 0.715-0.809 at 3-year and 0.719-0.742 at 5-year for HCC; AUC = 0.704-0.777 at 3-year and 0.721-0.735 at 5-year for LRE). Among patients without cirrhosis, mREACH-B predicted HCC (AUC $=0.803$ vs. $0.654-0.816$ at 3-year and 0.684 vs. 0.639-0.738 at 5-year) and LRE development (AUC $=0.734$ vs. 0.619-0.789 at 3-year and 0.674 vs. 0.626-0.729 at 5-year) similarly to PAGE-B, REACH-B, and LSM-HCC. mREACH-B appropriately predicted HCC and LRE development in patients with CHB and showed similar or superior accuracy to those of PAGE-B, REACH-B, and LSM-HCC.

\section{INTRODUCTION}

Chronic hepatitis B (CHB) virus infection is a leading cause of liver cirrhosis and hepatocellular carcinoma (HCC), especially in Asian countries, where chronic hepatitis B virus (HBV) infection is endemic [1, 2]. If HCC is diagnosed at an early stage during surveillance, the chance of a 'cure' and corresponding favorable long-term outcomes can be 
expected. Thus, risk stratification and the early detection of HCC are of great importance in patients with CHB.

To date, several risk prediction models including the REACH-B and PAGE-B models showing acceptable accuracy have been proposed [3]. Of these, the REACH-B model, which includes gender, age, alanine aminotransferase (ALT) level, hepatitis B e antigen (HBeAg) status, and HBV DNA level as variables, was proposed from an Asian multi-center study [3]. However, because REACH-B was established from the cohort of CHB patients without cirrhosis, it is not applicable to the whole spectrum of patients with $\mathrm{CHB}$, such as those with cirrhosis, who are at higher risk of HCC development and may benefit most from risk stratification. In addition, the prognostic accuracy of the PAGE-B model, which was established from Caucasian subjects with CHB [4], has not fully validated yet in Asian subjects.

Recently, based on the concept that detailed stratification according to fibrotic burden can be more prognostic [5], several liver stiffness (LS)-based risk prediction models such as the modified REACH-B (mREACH-B) and LSM-HCC models have been proposed. Although the prognostic accuracy of mREACH-B [6], which was established from REACH-B model by incorporating LS, assessed using transient elastography (TE) instead of HBV DNA, has been confirmed in several studies [7, 8], its external validation has yet to be performed. In addition, LSM-HCC model was recently proposed [9]. However, further validation of its prognostic accuracy and the comparison with mREACH-B are still required.

Thus, in this multi-center retrospective cohort study, we sought to validate the prognostic accuracy of mREACH-B in predicting the risk of HCC and liverrelated event (LRE) development in comparison with other risk prediction models, including PAGE-B, REACH-B, and LSM-HCC models, in patients with CHB.

\section{MATERIALS AND METHODS}

\section{Patients}

A total of 1,538 patients with CHB who underwent TE examinations at four tertiary institutions (Gangnam Severance Hospital, Dongsan Medical Center, Korea University Hospital, and Soonchunhyang University Bucheon Hospital) from 2006 to 2012 were recruited for this retrospective multi-center cohort study. CHB was defined as the persistent presence of the serum HBV surface antigen for $>6$ months.

Exclusion criteria were as follows: 1) TE examination failure (valid shot $=0$ ), 2) unreliable LS values, 3) HCC or LRE development $<6$ months after enrollment, 4) current or previous history of HCC, decompensation, or liver transplantation, 5) Child-Pugh class B or C, 6) co-infection with hepatitis C or HIV,
7) right-sided heart failure, 8) ascites or pregnancy, 9) significant alcohol consumption (> $40 \mathrm{~g} /$ daily), 10) significant medical comorbidities, and 11) insufficient data for risk model calculation (Supplementary Figure 1).

The study conformed to the ethical guidelines of the 1975 Declaration of Helsinki and was approved by the institutional review board of each institute. The requirement for written informed consent was waived due to the retrospective nature of the study.

\section{Follow-up}

Each patient was screened for HCC with ultrasonography at their initial visit. If no evidence of HCC was detected, patients were followed up with afetoprotein and ultrasonography every 3 or 6 months for HCC surveillance. Antiviral therapy (AVT) was started according to the guidelines of the Korean Association for the Study of the Liver [10]. During surveillance, HCC was diagnosed based on the guidelines of the American Association for the Study of Liver Diseases [1].

\section{Primary end-points}

The primary aim of this study was to evaluate the predictive value of risk prediction models for assessing the risks of HCC and LRE development. To avoid statistical repetition, we selected the earliest of LREs as a major event if a given patient experienced different types of LRE at different times. LRE included the development of HCC, decompensation, liver transplantation, and liver-related death.

\section{Assessment of liver stiffness using transient elastography}

The LS value was assessed using TE (FibroScan; EchoSens, Paris, France). LS values measured by experienced technicians or nurses ( $>500$ examinations) were expressed in kilopascals $(\mathrm{kPa})$. The detailed process for TE assessment has been described previously [1113]. The interquartile range (IQR) served as an index of the intrinsic variability in LS values and corresponded to the interval of LS results containing $50 \%$ of the valid measurements between the 25th and 75th percentiles. The median value of the successful measurements was regarded as representative of the LS value only if the IQR to median value ratio was $<30 \%$. In addition, the LS value with at least 10 valid measurements and a success rate $>$ $60 \%$ was considered reliable.

\section{Selection of risk prediction models for comparison}

First, the REACH-B model, from which mREACH-B was derived, was selected [14]. The 
mREACH-B score substitutes the LS value for the HBV DNA level in the REACH-B model [6]. Additionally, another LS-based LSM-HCC model was selected to compare its prognostic accuracy with mREACH-B [9]. The LSM-HCC score was generated from the LS values, age, serum albumin level, and HBV DNA level. Finally, we selected a recently proposed prediction model from Caucasian patients with CHB receiving AVT [15], named PAGE-B, which has age, gender, and platelet count, as constituent variables, because only one previous study validated PAGE-B in Asian patients with $\mathrm{CHB}$ with no comparison with the accuracy of LS-based models [4]. The detailed calculation methods are summarized in Supplementary Table 1.

\section{Statistical analysis}

Data are expressed as medians with IQRs or as $n(\%)$, as appropriate. Student's $t$-test (or the MannWhitney test) and the $\chi^{2}$ test (or Fisher's exact test) were used to compare the baseline characteristics of patients with and without liver cirrhosis at baseline, which was diagnosed based on ultrasonographic findings, including splenomegaly, blunt angle, and morphological changes (nodularity of liver surface, atrophy of the right lobe, hypertrophy of the left and caudate lobes, expansion of periportal spaces, and intrahepatic nodules). For subgroup analysis, high ALT level was defined as $>40 \mathrm{IU} / \mathrm{mL}$. Additionally, baseline characteristics of patients who developed HCC or LRE and those who did not were compared. Patients were censored at the time of first presentation of HCC or LRE according to the selection of end-points or at the last follow-up. The annual and cumulative incidence rates of $\mathrm{HCC}$ were calculated using the Kaplan-Meier method. To identify independent risk factors for HCC development, univariate and subsequent multivariate Cox proportional hazard regression analyses were conducted. Hazard ratios (HRs) and corresponding 95\% confidence intervals (CIs) are presented. The 3-, 5-, and 7-year cumulative incidences of HCC were assessed by calculating area under the curves (AUCs). The AUCs of the risk prediction models were compared between pairs using the method of Delong et al. A $P$ value $<0.05$ (two-tailed test) was considered to indicate statistical significance. All analyses were performed using the SPSS software (ver. 20.0; SPSS Inc., Chicago, IL, USA).

\section{RESULTS}

\section{Baseline characteristics}

After excluding 61 patients due to TE examination failure or unreliable LS values (drop-out rate due to TE, 4.0\%), 1,477 patients with a reliable LS value for calculating risk prediction models were selected. Then, a further 236 patients were excluded according to our exclusion criteria. Finally, 1,241 patients with CHB were included in this retrospective multi-center cohort study (Supplementary Figure 1).

Baseline characteristics at enrollment are summarized in Table 1. The median age of the patients (840 males, 401 females) was 49 years. In total, 301 (24.3\%) patients had liver cirrhosis and 557 (44.9\%) were receiving AVT at enrollment. The median LS value was $3.9 \mathrm{kPa}$. Additionally, the median PAGE-B value was 10 and those of the LS-based prediction models of LSM-HCC and mREACH-B were 10 and 8 , respectively. Among patients without liver cirrhosis, the median REACH-B value was 9 .

\section{Comparison between patients with and without liver cirrhosis}

When the study population was stratified into two groups, with and without liver cirrhosis (Table 1), age, the proportions of male gender, diabetes, and ongoing AVT, total bilirubin level, and LS value were significantly higher in patients with liver cirrhosis than in those without it, whereas serum albumin level, aspartate aminotransferase (AST) level, ALT level, the proportion of $\mathrm{HBeAg}$ positivity, HBV DNA level, and platelet count were significantly lower in patients with liver cirrhosis (all $P<0.05)$. PAGE-B, LSM-HCC, and mREACH-B values were significantly higher in patients with liver cirrhosis than in those without it (all $P<0.001$ ).

\section{HCC and LRE development}

The median follow-up period from enrollment was 77.4 (IQR 56.2-97.7) months. In total, 66 (5.3\%) and 83 (6.7\%) patients developed HCC and LRE, respectively. For patients who developed LRE, HCC mostly comprised LRE (64, 77.1\%), followed by decompensation $(n=17,20.5 \%)$ and liver-related death $(n=2,2.4 \%)$. The cumulative incidence rates at 3,5 , and 7 years were $2.4 \%$, $4.6 \%$, and $6.3 \%$ for HCC development and 3.0\%, 5.6\%, and $7.7 \%$ for LRE development, respectively (Figure 1).

When the study population was stratified according to liver cirrhosis, patients with liver cirrhosis experienced HCC and LRE development more frequently (1.1\%, $2.6 \%$, and $3.5 \%$ for HCC and $8.8 \%, 15.1 \%$, and $20.6 \%$ for LRE, respectively) than those without it $(0.7 \%, 2.0 \%$, and $2.5 \%$ for HCC and $7.7 \%, 12.5 \%$, and $17.8 \%$ for LRE, respectively; log-rank test, all $P<0.001$; Supplementary Figure 2).

\section{Comparisons between patients who developed HCC and LRE and those who did not}

Characteristics of the $66(5.3 \%)$ patients who developed HCC and the 1,174 (94.6\%) patients who did not and the $83(6.7 \%)$ patients who developed LRE 
and the $1,158(93.3 \%)$ patients who did not are shown in Table 2. The age and proportions of hypertension and liver cirrhosis, total bilirubin level, LS value, and the values of three prediction models (PAGE-B, LSM-HCC, and mREACH-B) were significantly higher in patients who developed HCC than in those who did not (all $P$ $<0.05$ ), whereas serum albumin level, ALT level, and platelet count were significantly lower in patients who developed HCC (all $P<0.05$ ). Similar findings were noted between patients who developed LRE and those who did not.

\section{Independent predictors of HCC and LRE development}

Independent predictors of $\mathrm{HCC}$ and LRE development were evaluated (Table 3). Univariate analyses identified age, liver cirrhosis, total bilirubin, serum albumin, platelet count, and LS value as significant predictors of HCC development, whereas age, liver cirrhosis, serum albumin, platelet count, and LS value were selected as significant predictors of LRE development (all $P<0.05$ ). According to a multivariate analysis, older age $(\mathrm{HR}=1.065)$, liver cirrhosis $(\mathrm{HR}=2.724)$, and higher $\mathrm{LS}$ value $(\mathrm{HR}=1.047)$ were independently associated with an increased risk of HCC development (all $P<0.05)$, whereas older age $(\mathrm{HR}=1.057)$, liver cirrhosis $(\mathrm{HR}=2.299)$, lower platelet count $(\mathrm{HR}=0.994)$, and higher LS value $(\mathrm{HR}=1.047)$ were independently associated with an increased risk of LRE development (all $P<0.05$ ).

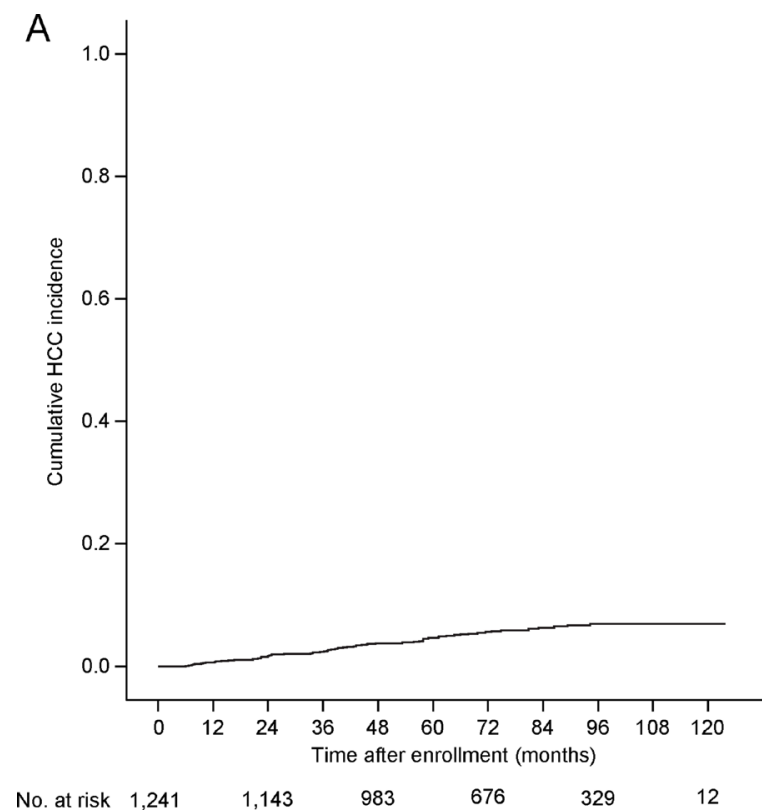

No. at risk 1,241

\section{Unadjusted hazard ratio of models in predicting HCC and LRE development}

The unadjusted HRs obtained from the prediction models are shown in Table 4. PAGE-B, LSM-HCC, and mREACH-B were significantly predictive of $\mathrm{HCC}$ and LRE development $(\mathrm{HR}=1.176-1.366$ for $\mathrm{HCC}$ and 1.109-1.325 for LRE; all $P<0.001)$. In patients without liver cirrhosis, PAGE-B, REACH-B, LSM$\mathrm{HCC}$, and mREACH-B were predictive of HCC and LRE development $(\mathrm{HR}=1.116-1.246$ for $\mathrm{HCC}$ and 1.087-1.214 for LRE; all $P<0.05)$, except for REACH-B in predicting LRE $(P=0.115)$. In patients with liver cirrhosis, PAGE-B, LSM-HCC, and mREACH-B were also predictive of HCC and LRE development $(\mathrm{HR}=1.074-1.248$ for $\mathrm{HCC}$ and 1.067-1.218 for LRE; all $P<0.05)$ except PAGE-B in predicting $\mathrm{HCC}(P=0.100)$.

\section{Predictive accuracy of models in predicting $\mathrm{HCC}$ and LRE development}

The performance of the risk prediction models in predicting $\mathrm{HCC}$ at 3, 5, and 7 years was calculated (Table 5, Figure 2). The accuracy of REACH-B was calculated only for patients without liver cirrhosis.

In the entire study population, the mREACH-B score showed higher performance in predicting $\mathrm{HCC}$ than those of the PAGE-B and LSM-HCC at 3 years $(\mathrm{AUC}=0.824$ vs. 0.715 and 0.809 , respectively), 5 years (AUC $=0.750$ vs. 0.719 and 0.742 , respectively), and 7 years (AUC $=0.770$ vs. 0.714 and 0.765 , respectively). However, a statistically significant difference was seen

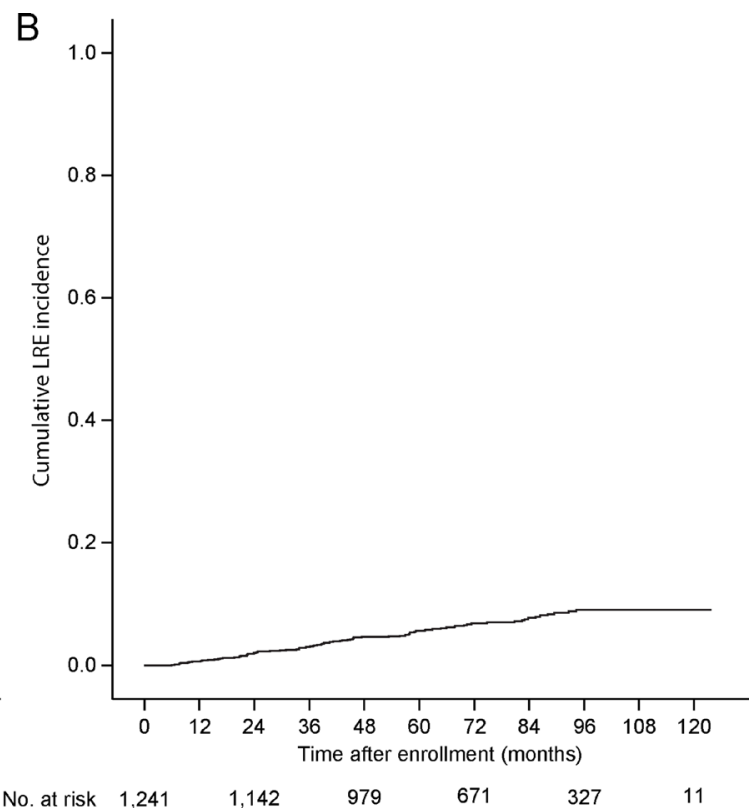

Figure 1: Cumulative incidence rates of HCC and LRE (Kaplan-Meier plot). The cumulative incidence rates of HCC at 3, 5 , and 7 years were $2.4 \%, 4.6 \%$, and $6.3 \%$, respectively (A), whereas those of LRE were $3.0 \%, 5.6 \%$, and $7.7 \%$, respectively (B). HCC, hepatocellular carcinoma; LRE, liver-related event. 
Table 1: Baseline characteristics of the study population $(n=1,241)$

\begin{tabular}{|c|c|c|c|c|}
\hline \multirow{2}{*}{ Variables } & \multirow{2}{*}{ All } & \multicolumn{2}{|c|}{ Liver cirrhosis } & \multirow[b]{2}{*}{$P$ value } \\
\hline & & Without $(940,75.7 \%)$ & With $(301,24.3 \%)$ & \\
\hline \multicolumn{5}{|l|}{ Demographic variables } \\
\hline Age, years & $49(40-57)$ & $47(38-55)$ & $54(48-61)$ & $<0.001$ \\
\hline Male gender & $840(67.7)$ & $617(65.6)$ & $223(74.1)$ & 0.007 \\
\hline BMI, $\mathrm{kg} / \mathrm{m}^{2}$ & $23.5(21.5-25.4)$ & $23.3(21.4-25.4)$ & $23.8(22.0-25.5)$ & 0.141 \\
\hline Diabetes & $92(7.4)$ & $55(5.9)$ & $37(12.3)$ & 0.001 \\
\hline Hypertension & $109(8.8)$ & $75(8.0)$ & $34(11.3)$ & 0.080 \\
\hline On-going AVT & $557(44.9)$ & $393(41.8)$ & $164(54.5)$ & $<0.001$ \\
\hline \multicolumn{5}{|l|}{ Laboratory variables } \\
\hline Total bilirubin, $\mathrm{mg} / \mathrm{dL}$ & $0.8(0.6-1.0)$ & $0.8(0.6-1.0)$ & $0.9(0.7-1.1)$ & $<0.001$ \\
\hline Serum albumin, $g / d L$ & $4.4(4.1-4.6)$ & $4.4(4.1-4.6)$ & $4.3(4.1-4.5)$ & $<0.001$ \\
\hline AST, IU/L & $32(23-53)$ & $30(22-52)$ & $36(27-53)$ & 0.004 \\
\hline ALT, IU/L & $35(22-71)$ & $35(21-79)$ & $35(24-56)$ & $<0.001$ \\
\hline AFP, $n g / m L$ & $3.10(2.10-5.00)$ & $2.90(2.00-4.50)$ & $3.95(2.55-7.00)$ & 0.537 \\
\hline HBeAg positivity & $562(45.3)$ & $452(48.1)$ & $110(36.5)$ & 0.001 \\
\hline HBV DNA, $\log _{10} I U / m L$ & $3.9(2.0-6.6)$ & $4.0(2.1-7.0)$ & $3.6(1.6-5.8)$ & 0.001 \\
\hline Platelet count, $10^{9} / \mathrm{L}$ & $185(142-228)$ & $198(161-237)$ & $136(101-179)$ & $<0.001$ \\
\hline Liver stiffness, $\mathrm{kPa}$ & $3.9(4.7-11.0)$ & $6.1(4.5-8.7)$ & $11.9(7.9-17.4)$ & $<0.001$ \\
\hline \multicolumn{5}{|l|}{ Risk prediction models } \\
\hline PAGE-B & $10(8-15)$ & $9(7-14)$ & $14(10-16)$ & $<0.001$ \\
\hline REACH-B & - & $9(7-11)$ & - & - \\
\hline LSM-HCC & $10(5-18)$ & $10(0-15)$ & $18(10-24)$ & $<0.001$ \\
\hline mREACH-B & $8(6-10)$ & $7(6-9)$ & $10(8-12)$ & $<0.001$ \\
\hline
\end{tabular}

Variables are expressed as median (interquartile range) or $\mathrm{n}(\%)$.

BMI, body mass index; AVT, antiviral therapy; AST, aspartate aminotransferase; ALT, alanine aminotransferase; AFP, alphafetoprotein; HBeAg, hepatitis B e antigen; $\mathrm{kPa}$, kilopascal.

only between mREACH-B and PAGE-B at 3 years $(P=$ $0.014)$.

When patients with normal ALT were selected ( $n=707,57.0 \%)$ to exclude the overestimating influence of high ALT on LS-based prediction models, AUC values of mREACH-B and LSM-HCC were higher than those of PAGE-B (AUC $=0.823-0.730$ for mREACH-B, $0.793-0.740$ for LSM-HCC vs. 0.734-0.699 for PAGE-B at 3-7 years), but the difference was not statistically significant (all $P>0.05$ ). However, among patients with high ALT levels $(n=534,43.0 \%)$, mREACH-B showed significantly higher AUC values than PAGE-B ( 0.826 vs. 0.696 at 3 years, $P=0.004$, and 0.818 vs. 0.736 at 7 years, $P=0.049$ ).

In the subgroup for patients without liver cirrhosis in whom REACH-B could be applied $(n=940,75.7 \%)$, PAGE-B, LSM-HCC, and mREACH-B showed higher AUC values than REACH-B (AUC $=0.738-0.693$ for PAGE-B, 0.816-0.706 for LSM-HCC, and 0.803-0.684 for mREACH-B vs. 0.654-0.639 for REACH-B), but the difference was not statistically significant (all $P>0.05$ ). Similar findings were seen in patients with liver cirrhosis $(n=301,24.3 \%)$.

When the performance of the risk prediction models in predicting LRE at 3,5, and 7 years was calculated (Supplementary Table 2, Supplementary Figure 3), overall trends for the predictive accuracy of prediction models were similar to those of $\mathrm{HCC}$ prediction.

\section{Predictive accuracy of models according to AVT status}

When the patients with on-going AVT at enrollment were selected $(n=557)$, mREACH-B showed similar or higher AUC value than PAGE-B and LSM-HCC models at 3 years ( 0.846 vs. 0.674 and 0.809$)$. At 5 and 7 years, AUC values of mREACH-B and LSM-HCC were similar (0.778 vs. 0.779 at 5 years; 0.788 vs. 0.789 at 7 years), but higher than those of PAGE-B model ( 0.688 at 5 years and 0.694 at 7 years). In addition, when the patients without on- 
going AVT were analyzed ( $n=684)$, mREACH-B showed similar or higher AUC values than those of PAGE-B and LSM-HCC models ( 0.764 vs. 0.741 and 0.716 at 3 years; 0.735 vs. 0.734 and 0.735 at 5 years; 0.784 vs. 0.727 and 0.741 at 7 years).

\section{Risk stratification according to mREACH-B}

We stratified the study population into four risk groups according to mREACH-B based on established cutoff values (Table 6) [8]. The cumulative incidence rates of HCC and LRE development were significantly different in low-risk versus low-intermediate-risk groups and between high-intermediate-risk and high-risk groups (all $P<0.05$, log-rank test), whereas they were statistically similar between low-intermediate-risk and high-intermediate risk groups $(P=0.270$, log-rank test; Supplementary

A

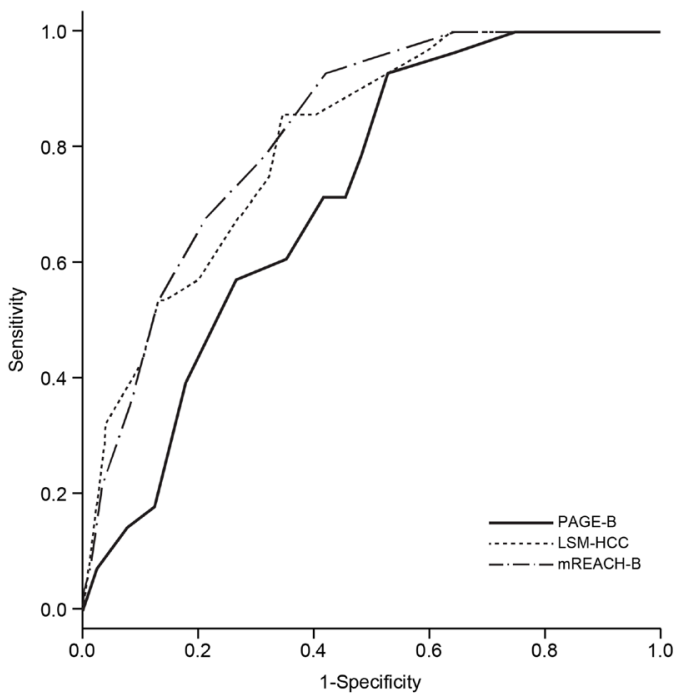

Figure 4). Similar trends were noted with regard to LRE development (Table 6, Supplementary Figure 4). However, when high- and low-intermediate risk groups were merged as an intermediate-risk group, there were significantly different rates of HCC and LRE development (data not shown).

\section{DISCUSSION}

An accurate assessment of the risk of HCC and LRE development is important for establishing individualized strategies of follow-up, intervention, and management, because this ultimately enables the extension of overall survival in patients with CHB. To address this, several risk prediction models for patients with $\mathrm{CHB}$ have been proposed $[6,9,14,15]$. In this study, we attempted to validate the prognostic accuracy of the mREACH-B model,

B

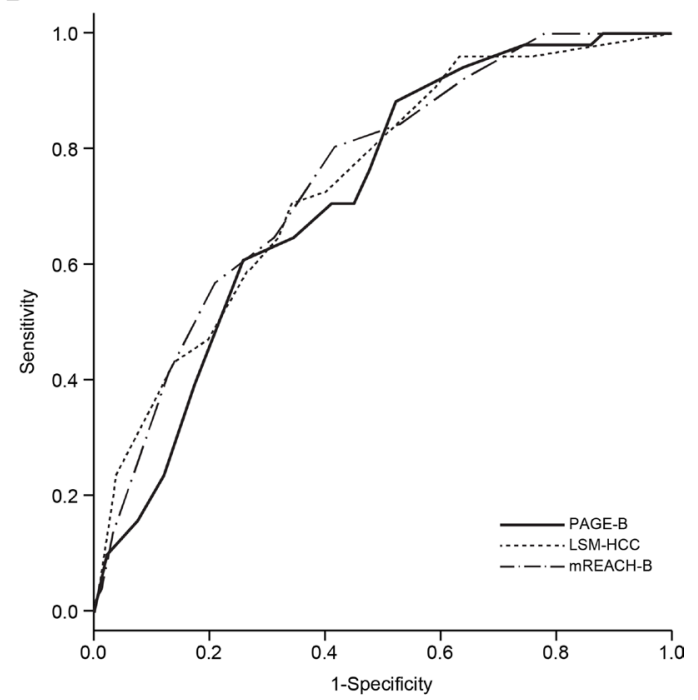

C

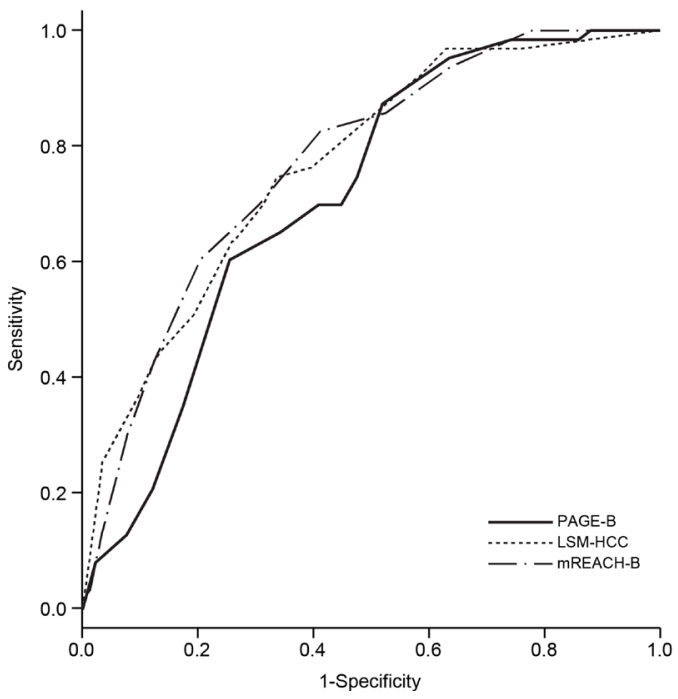

Figure 2: Receiver operating characteristic curves of the PAGE-B, LSM-HCC, and mREACH-B scores for the prediction of HCC development at 3 (A), 5 (B), and 7 years (C) in the entire study population. HCC, hepatocellular carcinoma. 
Table 2: Baseline characteristics of patients who developed HCC and LRE versus those who did not

\begin{tabular}{|c|c|c|c|c|c|c|}
\hline \multirow{3}{*}{ Variables } & \multicolumn{3}{|c|}{ Hepatocellular carcinoma } & \multicolumn{3}{|c|}{ Liver-related event } \\
\hline & Without & With & \multirow{2}{*}{$P$ value } & Without & With & \multirow{2}{*}{$P$ value } \\
\hline & $(n=1,174,94.6 \%)$ & $(n=66,5.3 \%)$ & & $(n=1,158,93.3 \%)$ & $(n=83,6.7 \%)$ & \\
\hline \multicolumn{7}{|l|}{ Demographic variables } \\
\hline Age, years & $49(40-56)$ & $58(51-64)$ & $<0.001$ & $49(39-56)$ & $57(50-64)$ & $<0.001$ \\
\hline Male gender & $789(67.2)$ & $50(75.8)$ & 0.176 & $780(67.4)$ & $60(72.3)$ & 0.396 \\
\hline Body mass index, $\mathrm{kg} / \mathrm{m}^{2}$ & $23.4(21.4-25.4)$ & $24.2(22.8-25.5)$ & 0.917 & $23.4(21.5-25.4)$ & $23.8(22.0-25.4)$ & 0.684 \\
\hline Diabetes & $85(7.2)$ & $7(10.6)$ & 0.329 & $83(7.2)$ & $9(10.8)$ & 0.198 \\
\hline Hypertension & $93(7.9)$ & $16(24.2)$ & $<0.001$ & $89(7.7)$ & $20(24.1)$ & $<0.001$ \\
\hline Liver cirrhosis & $255(21.7)$ & $46(69.7)$ & $<0.001$ & $246(21.2)$ & $55(66.3)$ & $<0.001$ \\
\hline On-going AVT & $522(44.5)$ & $34(51.5)$ & 0.309 & $520(44.9)$ & $37(44.6)$ & 0.999 \\
\hline \multicolumn{7}{|l|}{ Laboratory variables } \\
\hline Total bilirubin, mg/dL & $0.8(0.6-1.0)$ & $0.9(0.6-1.2)$ & 0.049 & $0.8(0.6-1.0)$ & $0.9(0.6-1.1)$ & 0.035 \\
\hline Serum albumin, $\mathrm{g} / \mathrm{dL}$ & $4.4(4.1-4.6)$ & $4.2(4.0-4.5)$ & 0.002 & $4.4(4.1-4.6)$ & $4.2(4.0-4.5)$ & 0.002 \\
\hline Aspartate aminotransferase, IU/L & $31(23-52)$ & $45(32-62)$ & 0.146 & $31(23-52)$ & $44(19-63)$ & 0.211 \\
\hline Alanine aminotransferase, IU/L & $35(21-71)$ & $40(28-63)$ & 0.001 & $35(22-71)$ & $40(25-64)$ & 0.001 \\
\hline Alpha-fetoprotein, $\mathrm{ng} / \mathrm{mL}$ & $3.10(2.10-4.88)$ & $4.70(2.50-10.15)$ & 0.749 & $3.10(2.10-6.70)$ & $4.10(2.33-9.43)$ & 0.704 \\
\hline $\mathrm{HBeAg}$ positivity & $536(45.7)$ & $26(39.4)$ & 0.374 & $528(45.6)$ & $34(41.0)$ & 0.427 \\
\hline HBV DNA, $\log \mathrm{IU} / \mathrm{mL}$ & $3.8(2.0-6.7)$ & $5.3(2.3-6.5)$ & 0.623 & $3.9(2.1-6.7)$ & $3.8(1.7-6.4)$ & 0.596 \\
\hline Platelet count, $10^{9} / \mathrm{L}$ & $190(144-229)$ & $125(1034-170)$ & $<0.001$ & $190(145-229)$ & $124(90-177)$ & $<0.001$ \\
\hline Liver stiffness, $\mathrm{kPa}$ & $6.8(4.8-10.5)$ & $12.2(8.4-21.2)$ & $<0.001$ & $6.8(4.8-10.4)$ & $12.0(7.9-20.9)$ & $<0.001$ \\
\hline \multicolumn{7}{|l|}{ Risk prediction models } \\
\hline PAGE-B & $10(7-15)$ & $15(11-16)$ & $<0.001$ & $10(7-15)$ & $15(10-16)$ & $<0.001$ \\
\hline LSM-HCC & $10(5-18)$ & $19(14-24)$ & $<0.001$ & $10(5-18)$ & $18(10-24)$ & $<0.001$ \\
\hline mREACH-B & $8(6-10)$ & $11(9-13)$ & $<0.001$ & $8(6-10)$ & $11(9-13)$ & $<0.001$ \\
\hline
\end{tabular}

Variables are expressed as median (interquartile range) or $\mathrm{n}(\%)$.

HCC, hepatocellular carcinoma; LRE, liver-related event; AVT, antiviral therapy; HBeAg, hepatitis B e antigen; kPa, kilopascal.

which is a modified version of the REACH-B model, and found that the mREACH-B model significantly predicted the risk of HCC and LRE development in the whole study population (AUC $\geq 0.750$ for $\mathrm{HCC}$ and $\geq 0.739$ for $\mathrm{LRE}$ at 3-7 years). Additionally, the mREACH-B model performed similarly or significantly better than the PAGE-B, REACH-B, and LSM-HCC models in our cohort.

Our study has several clinical implications. First, this study involved a large number of patients $(n=1,241)$ and a long follow-up period (median $>6$ years and maximum around 10 years), which may have increased the statistical power and reliability. We attempted to obtain a larger number of patients who achieved our primary endpoints during this long-term follow-up period $(n=66$, $5.3 \%$ for HCC and $n=83,6.7 \%$ for LRE). Additionally, this large sample size could facilitate stratification of the study population according to cirrhosis and ALT level. In the entire study population, the mREACH-B score showed significantly superior predictive value for HCC and LRE development at 3 years compared with that of PAGE-B (AUC $=0.824$ vs. 0.715 for $\mathrm{HCC}$ and 0.782 vs. 0.704 for LRE, all $P<0.05$ ), and similar predictive value to that of LSM-HCC at 3,5 , and 7 years. When a subgroup without cirrhosis was selected to investigate the accuracy of REACH-B, we found that the accuracy of mREACH-B was higher than that of the original REACH-B model ( $\mathrm{AUC}=0.803-0.684$ vs. $0.639-0.654$ for HCC and $0.734-0.674$ vs. $0.626-0.600$ for LRE). However, the difference was not statistically significant, probably due to the relatively small sample size of the participants and events in this subgroup without cirrhosis. Beyond these results, that the LS value was selected as an independent predictor of HCC and LRE development (both HR $=1.047$ ) and REACH-B did not show a significant unadjusted HR for predicting LRE may support the superiority of LS-based risk prediction models. Additionally, stratification according to ALT level revealed that the accuracy of mREACH-B was maintained at 3-7 years $(\mathrm{AUC}=0.723-0.792$ in normal ALT vs. $0.786-0.756$ in high ALT) and its similar or superior accuracy when compared to those of other models was also maintained, regardless of ALT level. In addition, in 
Table 3: Independent predictor of HCC and LRE development

\begin{tabular}{|c|c|c|c|c|c|c|c|c|}
\hline \multirow{3}{*}{ Variables } & \multicolumn{4}{|c|}{ Hepatocellular carcinoma } & \multicolumn{4}{|c|}{ Liver-related event } \\
\hline & \multirow{2}{*}{$\begin{array}{c}\text { Univariate } \\
P \text { value }\end{array}$} & \multicolumn{3}{|c|}{ Multivariate } & \multirow{2}{*}{$\begin{array}{c}\text { Univariate } \\
P \text { value }\end{array}$} & \multicolumn{3}{|c|}{ Multivariate } \\
\hline & & $\begin{array}{c}\text { Hazard } \\
\text { ratio }\end{array}$ & $95 \% \mathrm{CI}$ & $P$ value & & $\begin{array}{c}\text { Hazard } \\
\text { ratio }\end{array}$ & $95 \%$ CI & $P$ value \\
\hline \multicolumn{9}{|l|}{ Demographic variables } \\
\hline Age, years & $<0.001$ & 1.065 & $1.038-1.093$ & $<0.001$ & $<0.001$ & 1.057 & $1.034-1.081$ & $<0.001$ \\
\hline Male gender & 0.173 & - & - & - & 0.398 & & & \\
\hline Body mass index, $\mathrm{kg} / \mathrm{m}^{2}$ & 0.853 & - & - & - & 0.725 & & & \\
\hline Diabetes & 0.335 & - & - & - & 0.230 & & & \\
\hline Liver cirrhosis & $<0.001$ & 2.724 & $1.495-4.965$ & 0.001 & $<0.001$ & 2.299 & $1.362-3.881$ & 0.002 \\
\hline On-going AVT & 0.335 & - & - & - & 0.798 & & & \\
\hline \multicolumn{9}{|l|}{ Laboratory variables } \\
\hline Total bilirubin, mg/dL & 0.021 & 1.369 & $0.768-2.437$ & 0.287 & 0.052 & & & \\
\hline Serum albumin, $\mathrm{g} / \mathrm{dL}$ & 0.001 & 1.054 & $0.484-2.295$ & 0.895 & $<0.001$ & 1.135 & $0.567-2.271$ & 0.720 \\
\hline Aspartate aminotransferase, IU/L & 0.582 & - & - & - & 0.999 & & & \\
\hline Alanine aminotransferase, IU/L & 0.163 & - & - & - & 0.140 & & & \\
\hline Alpha-fetoprotein, $\mathrm{ng} / \mathrm{mL}$ & 0.733 & - & - & - & 0.650 & & & \\
\hline $\mathrm{HBe} A g$ positivity & 0.269 & - & - & - & 0.351 & & & \\
\hline HBV DNA, $\log \mathrm{IU} / \mathrm{mL}$ & 0.693 & - & - & - & 0.508 & & & \\
\hline Platelet count, 109/L & $<0.001$ & 0.996 & $0.991-1.001$ & 0.144 & $<0.001$ & 0.994 & $0.989-0.999$ & 0.012 \\
\hline Liver stiffness, $\mathbf{k P a}$ & $<0.001$ & 1.047 & $1.018-1.077$ & 0.002 & $<0.001$ & 1.047 & $1.021-1.074$ & $<0.001$ \\
\hline
\end{tabular}

Table 4: Unadjusted hazard ratios of prediction models

\begin{tabular}{lcccccc}
\hline \multirow{2}{*}{ Models } & \multicolumn{3}{c}{ Hepatocellular carcinoma } & \multicolumn{3}{c}{ Liver-related event } \\
\cline { 2 - 7 } All & Hazard ratio & $\mathbf{9 5 \%}$ CI & P value & Hazard ratio & $\mathbf{9 5 \%}$ CI & P value \\
PAGE-B & 1.176 & $1.107-1.250$ & $<0.001$ & 1.158 & $1.098-1.220$ & $<0.001$ \\
REACH-B & - & - & - & - & - & - \\
LSM-HCC & 1.125 & $1.091-1.160$ & $<0.001$ & 1.109 & $1.080-1.138$ & $<0.001$ \\
mREACH-B & 1.366 & $1.256-1.486$ & $<0.001$ & 1.325 & $1.231-1.426$ & $<0.001$ \\
Without cirrhosis & & & & & & \\
PAGE-B & 1.174 & $1.057-1.303$ & 0.003 & 1.104 & $1.017-1.198$ & 0.018 \\
REACH-B & 1.219 & $1.048-1.417$ & 0.010 & 1.103 & $0.976-1.247$ & 0.115 \\
LSM-HCC & 1.116 & $1.057-1.178$ & $<0.001$ & 1.087 & $1.039-1.137$ & $<0.001$ \\
mREACH-B & 1.246 & $1.074-1.445$ & 0.004 & 1.214 & $1.071-1.376$ & 0.002 \\
With cirrhosis & & & & & & 0.026 \\
PAGE-B & 1.069 & $0.987-1.158$ & 0.100 & 1.090 & $1.011-1.176$ & 0.026 \\
REACH-B & - & - & - & - & - & - \\
LSM-HCC & 1.074 & $1.033-1.117$ & $<0.001$ & 1.067 & $1.030-1.105$ & $<0.001$ \\
mREACH-B & 1.248 & $1.113-1.399$ & $<0.001$ & 1.218 & $1.101-1.349$ & $<0.001$ \\
\hline
\end{tabular}

CI, confidence interval. 
Table 5: Comparison of the prognostic accuracy of risk prediction models for HCC development

\begin{tabular}{|c|c|c|c|c|c|c|c|c|c|}
\hline \multirow{2}{*}{$\begin{array}{l}\text { Study groups } \\
\text { Prediction models }\end{array}$} & \multicolumn{3}{|c|}{ At 3 years } & \multicolumn{3}{|c|}{ At 5 years } & \multicolumn{3}{|c|}{ At 7 years } \\
\hline & AUC & $95 \% \mathrm{CI}$ & $P$ value ${ }^{1}$ & AUC & $95 \%$ CI & $P$ value ${ }^{1}$ & AUC & $95 \%$ CI & $P$ value \\
\hline \multicolumn{10}{|l|}{ All $(n=1,241)$} \\
\hline PAGE-B & $0.715^{* *}$ & $0.644-0.787$ & 0.014 & $0.719^{* *}$ & $0.659-0.779$ & 0.445 & $0.714^{* *}$ & $0.661-0.766$ & 0.111 \\
\hline REACH-B & - & - & - & - & - & - & - & - & - \\
\hline LSM-HCC & $0.809^{* *}$ & $0.742-0.876$ & 0.444 & $0.742^{* *}$ & $0.677-0.809$ & 0.639 & $0.765^{* *}$ & $0.709-0.821$ & 0.781 \\
\hline mREACH-B & $0.824^{* *}$ & $0.765-0.884$ & - & $0.750^{* *}$ & $0.689-0.811$ & - & $0.770^{* *}$ & $0.717-0.823$ & - \\
\hline \multicolumn{10}{|c|}{ With normal ALT $(n=707)$} \\
\hline PAGE-B & $0.734^{*}$ & $0.652-0.817$ & 0.174 & $0.704^{* *}$ & $0.630-0.778$ & 0.541 & $0.699^{* *}$ & $0.628-0.770$ & 0.541 \\
\hline REACH-B & - & - & - & - & - & - & - & - & - \\
\hline LSM-HCC & $0.793^{* *}$ & $0.652-0.884$ & 0.312 & $0.742^{* *}$ & $0.663-0.822$ & 0.842 & $0.740^{* *}$ & $0.665-0.816$ & 0.687 \\
\hline mREACH-B & $0.823^{* *}$ & $0.740-0.906$ & - & $0.737^{* *}$ & $0.658-0.816$ & - & $0.730^{* *}$ & $0.654-0.807$ & - \\
\hline \multicolumn{10}{|c|}{ With high ALT $(n=534)$} \\
\hline PAGE-B & $0.696^{*}$ & $0.577-0.814$ & 0.004 & $0.737^{* *}$ & $0.638-0.836$ & 0.359 & $0.736^{* *}$ & $0.658-0.813$ & 0.049 \\
\hline REACH-B & - & - & - & - & - & - & - & - & - \\
\hline LSM-HCC & $0.837^{* *}$ & $0.744-0.929$ & 0.658 & $0.766^{* *}$ & $0.660-0.872$ & 0.401 & $0.801^{* *}$ & $0.723-0.879$ & 0.447 \\
\hline mREACH-B & $0.826^{* *}$ & $0.744-0.907$ & - & $0.786^{* *}$ & $0.701-0.871$ & - & $0.818^{* *}$ & $0.755-0.880$ & - \\
\hline \multicolumn{10}{|c|}{ Without cirrhosis $(n=940)$} \\
\hline PAGE-B & 0.693 & $0.542-0.843$ & 0.399 & $0.738^{* *}$ & $0.648-0.829$ & 0.535 & $0.721^{*}$ & $0.637-0.805$ & 0.879 \\
\hline REACH-B & 0.654 & $0.477-0.831$ & 0.280 & 0.639 & $0.506-0.773$ & 0.600 & $0.653^{*}$ & $0.535-0.772$ & 0.221 \\
\hline LSM-HCC & $0.816^{*}$ & $0.704-0.928$ & 0.734 & $0.706^{*}$ & $0.577-0.836$ & 0.556 & $0.725^{*}$ & $0.609-0.840$ & 0.507 \\
\hline mREACH-B & $0.803^{*}$ & $0.667-0.940$ & - & $0.684^{*}$ & $0.564-0.804$ & - & $0.691^{*}$ & $0.584-0.798$ & - \\
\hline \multicolumn{10}{|c|}{ With cirrhosis $(n=301)$} \\
\hline PAGE-B & 0.584 & $0.475-0.694$ & 0.083 & 0.591 & $0.494-0.689$ & 0.374 & 0.582 & $0.497-0.667$ & 0.074 \\
\hline REACH-B & - & - & - & - & - & - & - & - & - \\
\hline LSM-HCC & $0.686^{*}$ & $0.574-0.798$ & 0.643 & $0.630^{*}$ & $0.533-0.727$ & 0.602 & $0.659^{*}$ & $0.575-0.743$ & 0.483 \\
\hline mREACH-B & $0.702^{*}$ & $0.598-0.805$ & - & $0.644^{*}$ & $0.554-0.735$ & - & $0.677^{* *}$ & $0.598-0.755$ & - \\
\hline
\end{tabular}

${ }^{1} P$ value indicates the comparison between mREACH-B and other prediction models. AUC ${ }^{*}$ and ${ }^{* *}$ indicate $P$ value of $<0.05$ and $<0.001$, respectively. HCC, hepatocellular carcinoma; AUC, area under curve; CI, confidence interval; ALT, alanine aminotransferase.

spite of well-known confounding influence of high ALT level on LS [16], the overall prognostic accuracy of LSbased risk models was slightly higher in the subgroup with high ALT level than that in the subgroup with normal ALT. Although the exact reasons are unclear, the relatively low ALT level of our study population (median 35 IU/L) and the high proportion of patients with on-going AVT at enrollment (around 45\%) might have attenuated the influence of high ALT level. Thus, the influence of a high ALT level on LS-based risk prediction models should be investigated further [17].

Second, the mREACH-B model showed statistically similar accuracy in predicting HCC and LRE development to the LSM-HCC model in the entire study population and various subgroups, although previous studies showed the superior accuracy of mREACH-B [7, 8]. Because LSM-HCC included HBV DNA level, with diminished importance due to AVT [5], and mREACH-B was established based on the empirical weight allocation on LS stratification, the accuracy of LS-based prediction models may be altered according to the proportion of patients with on-going AVT and the different weight allocation strategy of the mREACH-B model for patients with high fibrotic burden. Further studies seem required to compare the predictive accuracy of these two LS-based risk models.

Third, we could compare the accuracy of PAGE-B with those of LS-based models. Although the PAGE-B model was recently proposed for Caucasian patients with CHB receiving AVT [15], only one validation study is currently available [4]; it concluded that the accuracy of PAGE-B was similar to that of the CU-HCC and GAG-HCC models, but significantly higher than that of the REACH-B model. In our study, AUC values of PAGE-B were also higher than those of REACH-B in predicting HCC and LRE development, but lower than those of LS-based models, although the differences were not statistically significant. Although the reason for this remains unclear, it may be explained in part by the platelet count, which is an important variable in the PAGE-B model, but was not selected as an independent predictor of HCC development $(P=0.144)$. Additionally, because the PAGE-B model was established from a cohort with AVT, 


\section{Table 6: Cumulative incidence rates of HCC and LRE development according to previously known}

cut-off values with $\mathrm{mREACH}-\mathrm{B}$

\begin{tabular}{lllll}
\hline $\begin{array}{l}\text { End-point } \\
\text { Risk groups }\end{array}$ & 3 years (\%) & $\mathbf{5}$ years (\%) & $\mathbf{7}$ years (\%) & $\boldsymbol{P}_{\text {value }}$ \\
\hline Hepatocellular carcinoma & & & & \\
Low-risk $<6(n=262,21.2 \%)$ & 0 & 0 & 0 & 0.005 \\
Low-intermediate-risk 6-7 $(n=306,24.6 \%)$ & 0.3 & 3.2 & 3.7 & 0.270 \\
High-intermediate-risk 8-10 $(n=394,31.7 \%)$ & 2.1 & 3.9 & 4.7 & $<0.001$ \\
High-risk $>10(n=279,22.5 \%)$ & 7.2 & 11.6 & 17 & - \\
Liver-related event & & & & \\
Low-risk $<6(n=262,21.2 \%)$ & 0.4 & 0.4 & 0.4 & 0.005 \\
Low-intermediate-risk 6-7 $(n=306,24.6 \%)$ & 1.0 & 3.9 & 4.4 & 0.152 \\
High-intermediate-risk $8-10(n=394,31.7 \%)$ & 3.6 & 4.8 & 7.2 & $<0.001$ \\
High-risk $>10(n=279,22.5 \%)$ & 8.0 & 13.6 & 19.8 & - \\
\hline
\end{tabular}

$P$ value ${ }^{1}$ indicates the comparison with adjacent higher risk group.

HCC, hepatocellular carcinoma; LRE, liver-related event.

its direct application in our cohort with mixed AVT status might have lessened the accuracy of the PAGE-B model.

Fourth, we tested not only the accuracy of risk prediction models for HCC development, but also for the comprehensive end-point of LRE, and found that all prediction models significantly predicted LRE development, except the REACH-B model $(P=0.115)$. Although HCC represented most LRE (77.1\%), the AUC values of prediction models became slightly lower when LRE was used as an end-point. Because the PAGE-B, REACH-B, and LSM-HCC models were established to predict HCC development, further validation studies on the applicability of the models in predicting LRE should be conducted.

We are aware of several other issues that should be taken into consideration. First, the study population was derived retrospectively from tertiary academic institutions, which might have resulted in the relatively high proportion of patients with liver cirrhosis (24.4\%). In addition, our definition of chronic hepatitis B might include HBV carriers rather than active hepatitis. Thus, our findings should be validated in future prospective studies involving the full spectrum of HBV disease in a community-based setting. Second, in some subgroups, the accuracy of the mREACH-B model was higher than those of other risk models. However, because the overall accuracy of the mREACH-B model in the entire population was not so prominent, we could not strongly insist the prognostic superiority of the mREACH-B score. Further validation studies are warranted to resolve this issue. Third, although all the technicians and nurses in our multi-center study who performed TE had sufficient experience [18], interinstitutional variability might have confounded our results. Additionally, the drop-out rates due to LS measurement failure or unreliable LS values (4.0\%) were significantly lower than in a European study [19]. However, this failure rate seems similar to those of other Asian studies (from $1.1 \%$ to $3.5 \%$ ) [20], probably due to the lower body mass index (median $23.5 \mathrm{~kg} / \mathrm{m}^{2}$ ) in our study. Moreover, during TE examinations, the skin capsular distance was not measured. Because this thickness can influence the detection of advanced fibrosis by TE [21], future studies should take this issue into considerations. Fourth, the risks of HCC and LRE development can change, because clinical and laboratory variables, such as fibrotic burden (liver stiffness and liver cirrhosis), ALT levels, HBeAg status, and HBV DNA levels, can change due to prolonged AVT, especially in this era of potent antiviral agents. Thus, our next multi-center study would investigate the impact of AVT on risk prediction models and the optimal cutoffs which might help to triage patients into different surveillance strategies. Finally, a reduction in LS value during AVT has been reported to be a favorable prognostic factor [22]. Thus, dynamic risk assessment using the risk prediction models is important. However, because we focused first on validating the accuracy of mREACH-B, in comparison with other prediction models, based on baseline characteristics, the clinical implication of dynamic changes in risk prediction models was not investigated in this study. Further well-designed studies with serial assessments of risk prediction models, which can provide more relevant clinical information and validate our results, should be performed.

In conclusion, the mREACH-B model appropriately predicted HCC and LRE development in patients with $\mathrm{CHB}$ and showed similar or superior accuracy to PAGE-B, REACH-B, and LSM-HCC. However, further validation studies are needed to investigate whether to incorporate mREACH-B into current surveillance strategies and its feasibility in dynamic assessments in patients with CHB. 


\section{Abbreviations}

CHB, chronic hepatitis B; HCC, hepatocellular carcinoma; HBV, hepatitis B virus; AVT, antiviral therapy; AUC, area under curve; ALT, alanine aminotransferase; $\mathrm{HBeAg}$, hepatitis B e antigen; mREACH-B, modified REACH-B; LS, liver stiffness; TE, transient elastography; LRE, liver-related event; HR, hazard ratio; CIs, confidence intervals; AST, aspartate aminotransferase.

\section{ACKNOWLEDGMENTS}

The authors are grateful to Dong-Su Jang, (Medical Illustrator, Medical Research Support Section, Yonsei University College of Medicine, Seoul, Republic of Korea) for his help with the figures.

\section{CONFLICTS OF INTEREST}

None to declare for all authors.

\section{FUNDING}

This study was supported by the Research Fund of the Korean Association for the Study of the Liver and the Basic Science Research Program through the National Research Foundation of Korea funded by the Ministry of Science, ICT \& Future Planning (2016R1A1A1A05005138). The funders had no role in the study design, data collection and analysis, decision to publish, or preparation of the manuscript.

\section{REFERENCES}

1. Bruix J, Sherman M, and American Association for the Study of Liver Diseases. Management of hepatocellular carcinoma: an update. Hepatology. 2011; 53:1020-22. https://doi.org/10.1002/hep.24199.

2. Kim BK, Han KH, Ahn SH. Prevention of hepatocellular carcinoma in patients with chronic hepatitis B virus infection. Oncology. 2011 (Suppl 1); 81:41-49. https://doi. org/10.1159/000333258.

3. Lee HW, Ahn SH. Prediction models of hepatocellular carcinoma development in chronic hepatitis B patients. World J Gastroenterol. 2016; 22:8314-21. https://doi. org/10.3748/wjg.v22.i37.8314.

4. Kim MN, Hwang SG, Rim KS, Kim BK, Park JY, Kim DY, Ahn SH, Han KH, Kim SU. Validation of PAGE-B model in Asian chronic hepatitis B patients receiving entecavir or tenofovir. Liver Int. 2017; 37:1788-95. https://doi. org/10.1111/liv.13450.

5. Jung KS, Kim SU, Song K, Park JY, Kim DY, Ahn SH, Kim BK, Han KH. Validation of hepatitis B virus-related hepatocellular carcinoma prediction models in the era of antiviral therapy. Hepatology. 2015; 62:1757-66. https:// doi.org/10.1002/hep. 28115 .
6. Lee HW, Yoo EJ, Kim BK, Kim SU, Park JY, Kim DY, Ahn $\mathrm{SH}$, Han KH. Prediction of development of liver-related events by transient elastography in hepatitis B patients with complete virological response on antiviral therapy. Am J Gastroenterol. 2014; 109:1241-49. https://doi.org/10.1038/ ajg.2014.157.

7. Kim MN, Kim SU, Kim BK, Park JY, Kim DY, Ahn $\mathrm{SH}$, Song KJ, Park YN, Han KH. Increased risk of hepatocellular carcinoma in chronic hepatitis B patients with transient elastography-defined subclinical cirrhosis. Hepatology. 2015; 61:1851-59. https://doi.org/10.1002/ hep. 27735.

8. Jeon MY, Lee HW, Kim SU, Heo JY, Han S, Kim BK, Park JY, Kim DY, Ahn SH, Han KH. Subcirrhotic liver stiffness by FibroScan correlates with lower risk of hepatocellular carcinoma in patients with HBV-related cirrhosis. Hepatol Int. 2017; 11:268-76. https://doi.org/10.1007/s12072-0179789-y.

9. Wong GL, Chan HL, Wong CK, Leung C, Chan CY, Ho PP, Chung VC, Chan ZC, Tse YK, Chim AM, Lau TK, Wong VW. Liver stiffness-based optimization of hepatocellular carcinoma risk score in patients with chronic hepatitis B. J Hepatol. 2014; 60:339-45. https://doi.org/10.1016/j. jhep.2013.09.029.

10. Korean Association for the Study of the Liver. KASL clinical practice guidelines: management of chronic hepatitis B. Clin Mol Hepatol. 2016; 22:18-75. https://doi. org/10.3350/cmh.2016.22.1.18.

11. Seo YS, Kim MY, Kim SU, Hyun BS, Jang JY, Lee JW, Lee JI, Suh SJ, Park SY, Park H, Jung EU, Kim BS, Kim IH, et al, and Korean Transient Elastography Study Group. Accuracy of transient elastography in assessing liver fibrosis in chronic viral hepatitis: A multicentre, retrospective study. Liver Int. 2015; 35:2246-55. https://doi.org/10.1111/ liv. 12808.

12. Kim SU, Song D, Heo JH, Yoo J, Kim BK, Park JY, Kim DY, Ahn SH, Kim KJ, Han KH, Kim YD. Liver fibrosis assessed with transient elastography is an independent risk factor for ischemic stroke. Atherosclerosis. 2017; 260:15662. https://doi.org/10.1016/j.atherosclerosis.2017.02.005.

13. Kim HS, Kim BK, Kim SU, Park JY, Kim DY, Song KJ, Park JW, Kim YJ, Baatarkhuu O, Han KH, Ahn SH. Association between level of fibrosis, rather than antiviral regimen, and outcomes of patients with chronic hepatitis B. Clin Gastroenterol Hepatol. 2016; 14:1647-1656.e6. https:// doi.org/10.1016/j.cgh.2016.05.039.

14. Yang HI, Yuen MF, Chan HL, Han KH, Chen PJ, Kim DY, Ahn SH, Chen CJ, Wong VW, Seto WK, and REACH-B Working Group. Risk estimation for hepatocellular carcinoma in chronic hepatitis B (REACH-B): development and validation of a predictive score. Lancet Oncol. 2011; 12:568-74. https://doi.org/10.1016/S1470-2045(11)70077-8.

15. Papatheodoridis G, Dalekos G, Sypsa V, Yurdaydin C, Buti M, Goulis J, Calleja JL, Chi H, Manolakopoulos S, Mangia G, Gatselis N, Keskin O, Savvidou S, et al. 
PAGE-B predicts the risk of developing hepatocellular carcinoma in Caucasians with chronic hepatitis B on 5-year antiviral therapy. J Hepatol. 2016; 64:800-06. https://doi. org/10.1016/j.jhep.2015.11.035.

16. Kim SU, Kim DY, Park JY, Lee JH, Ahn SH, Kim JK, Paik YH, Lee KS, Chon CY, Choi EH, Song KJ, Park YN, Han KH. How can we enhance the performance of liver stiffness measurement using FibroScan in diagnosing liver cirrhosis in patients with chronic hepatitis B? J Clin Gastroenterol. 2010; 44:66-71. https://doi.org/10.1097/ MCG.0b013e3181a95c7f.

17. Cho HJ, Seo YS, Lee KG, Hyun JJ, An H, Keum B, Kim JH, Yim HJ, Jeen YT, Lee HS, Chun HJ, Um SH, Kim CD, Ryu HS. Serum aminotransferase levels instead of etiology affects the accuracy of transient elastography in chronic viral hepatitis patients. J Gastroenterol Hepatol. 2011; 26:492-500. https://doi.org/10.1111/j.14401746.2010.06419.x.

18. Boursier J, Konate A, Guilluy M, Gorea G, Sawadogo A, Quemener E, Oberti F, Reaud S, Hubert-Fouchard I, Dib N, Calès P. Learning curve and interobserver reproducibility evaluation of liver stiffness measurement by transient elastography. Eur J Gastroenterol Hepatol. 2008; 20:693701. https://doi.org/10.1097/MEG.0b013e3282f51992.
19. Castéra L, Foucher J, Bernard PH, Carvalho F, Allaix D, Merrouche W, Couzigou P, de Lédinghen V. Pitfalls of liver stiffness measurement: a 5-year prospective study of 13,369 examinations. Hepatology. 2010; 51:828-35.

20. Kim SU, Han KH, Ahn SH. Transient elastography in chronic hepatitis B: an Asian perspective. World J Gastroenterol. 2010; 16:5173-80. https://doi.org/10.3748/ wjg.v16.i41.5173.

21. Kumagai E, Korenaga K, Korenaga M, Imamura M, Ueyama M, Aoki Y, Sugiyama M, Murata K, Masaki N, Kanto T, Mizokami M, Watanabe S. Appropriate use of virtual touch quantification and FibroScan $\mathrm{M}$ and XL probes according to the skin capsular distance. J Gastroenterol. 2016; 51:496-505. https://doi.org/10.1007/s00535-0151127-3.

22. Kim JH, Kim MN, Han KH, Kim SU. Clinical application of transient elastography in patients with chronic viral hepatitis receiving antiviral treatment. Liver Int. 2015; 35:1103-15. https://doi.org/10.1111/liv.12628. 